\title{
A Complex Integrable Hierarchy and Its Hamiltonian Structure for Integrable Couplings of WKI Soliton Hierarchy
}

\author{
Fajun Yu, Shuo Feng, and Yanyu Zhao \\ College of Maths and Systematic Science, Shenyang Normal University, Shenyang 110034, China \\ Correspondence should be addressed to Fajun Yu; fajun_yu@163.com
}

Received 14 April 2014; Accepted 4 June 2014; Published 26 June 2014

Academic Editor: Huanhe Dong

Copyright ( 2014 Fajun Yu et al. This is an open access article distributed under the Creative Commons Attribution License, which permits unrestricted use, distribution, and reproduction in any medium, provided the original work is properly cited.

\begin{abstract}
We generate complex integrable couplings from zero curvature equations associated with matrix spectral problems in this paper. A direct application to the WKI spectral problem leads to a novel soliton equation hierarchy of integrable coupling system; then we consider the Hamiltonian structure of the integrable coupling system. We select the $\bar{U}, \bar{V}$ and generate the nonlinear composite parts, which generate new extended WKI integrable couplings. It is also indicated that the method of block matrix is an efficient and straightforward way to construct the integrable coupling system.
\end{abstract}

\section{Introduction}

In what follows, we say that a system of partial differential equations (PDEs) or ordinary differential equations (PDEs) is integrable if it has infinitely many symmetries which are functionally independent on the jet spaces and Liouville integrable if it is Hamiltonian and possesses infinitely many conserved densities being functionally independent on the jet spaces. However, how to search for the new equation hierarchy and its Hamiltonian structure is an important work.

Integrable coupling system is an interesting and important topic in soliton theory [1-23]. Many coupled equations appear in practical problems, such as biochemistry, physics, and mechanics. For example, in order to systematically describe a kind of biochemistry model, Prigogine and Lefever proposed a coupled mathematical model in [1], which describes a biology-chemistry model; the well-known shallow water wave mathematics model [2]; and coupled KdV model [3], so that many integrable coupled mathematical models can solve practical problems [4].

A few ways to construct integrable couplings are presented by using perturbations $[5,6]$, enlarging spectral problems $[7,8]$, and creating new loop algebras $[9,10]$. The study of integrable couplings not only generalizes the symmetry problem, but also provides clues towards complete classification of integrable systems. The first point has been clearly presented and analyzed carefully in $[5,6,11,12]$.
Professors Ma and Fuchssteiner first proposed perturbation method for establishing integrable couplings [5]. Professors $\mathrm{Y}$. Zhang and $\mathrm{H}$. Zhang presented the enlarged Lie algebra method to obtain integrable couplings [9]. On the other hand, the theory of integrable couplings brings other interesting results such as Lax pairs of block form and several spectral parameters $[13,14]$, integrable constrained flows with higher multiplicity [15], local bi-Hamiltonian structures in higher dimensions [11], and hereditary recursion operators of higher order $[5,16]$. On the other hand, Ma et al. once used semidirect sums of Lie algebras to obtain the discrete integrable coupling system in [17].

The integrable coupling system is presented by using Virasoro symmetry algebra. In details, let $u_{t}=K(u)$ be a known integrable system; the following system $\left\{u_{t}=\right.$ $\left.K(u), v_{t}=S(u, v)\right\}$ is called integrable coupling of the system $u_{t}=K(u)$, if $v_{t}=S(u, v)$ is also integrable and $S(u, v)$ contains explicitly $u$ or $u$-derivatives with respect to $x$.

In recent years, it is becoming increasingly important to search for a new method to construct the integrable couplings of soliton equation hierarchy. As everyone knows that $\mathrm{Tu}$ method is a power tool to generate the soliton hierarchy and Hamiltonian functions. In this paper, we will use Ma's constructing method to produce new miraculous integrable couplings of WKI soliton hierarchy and its Hamiltonian structure with Lax pairs $\bar{U}$ and $\bar{V}[6]$. 


\section{The WKI Soliton Hierarchy and Its Hamiltonian Structure}

The traditional spatial spectral problem for the WKI hierarchy is given with $g=\operatorname{sl}(2, R)[19]$ :

$$
\begin{gathered}
\psi_{x}=[U, \psi], \quad \lambda_{t}=0, \\
U=U[u, \lambda]=\left(\begin{array}{cc}
-i \lambda & p \lambda \\
q \lambda & i \lambda
\end{array}\right), \quad V=\left(\begin{array}{cc}
-i \lambda a & \lambda p a+b_{x} \\
\lambda q a+c_{x} & i \lambda a
\end{array}\right) .
\end{gathered}
$$

We solve the stationary zero-curvature equation

$$
V_{x}=[U, V]
$$

and acquire the following system:

$$
\begin{gathered}
-i a_{x}=p c_{x}-q b_{x}, \\
\lambda p a+b_{x}=-2 i \lambda b, \\
\lambda q a+c_{x}=2 i \lambda c .
\end{gathered}
$$

Letting $a=\sum_{m=1}^{\infty} a_{m} \lambda^{-m}, b=\sum_{m=1}^{\infty} b_{m} \lambda^{-m}$, and $c=$ $\sum_{m=1}^{\infty} c_{m} \lambda^{-m}$, then we can obtain the recursive equations:

$$
\begin{gathered}
-i a_{m x}=p c_{m x}-q b_{m x}, \\
p a_{m+1}+b_{m x}=-2 i b_{m+1}, \\
q a_{m+1}+c_{m x}=2 i c_{m+1} .
\end{gathered}
$$

According to system (4), a new system is given as follows:

$$
\begin{gathered}
a_{m+1}=\frac{1}{2 \sqrt{1-p q}} \partial^{-1}\left(\frac{q}{\sqrt{1-p q}} b_{m x x}+\frac{p}{\sqrt{1-p q}} c_{m x x}\right), \\
b_{m+1}=\frac{i p}{2} a_{m+1}+\frac{i}{2} b_{m x}, \\
c_{m+1}=-\frac{i q}{2} a_{m+1}-\frac{i}{2} c_{m x},
\end{gathered}
$$

and the initial values satisfy the contents:

$$
\begin{gathered}
(2-2 p q) a_{0 x}-(p q)_{x} a_{0}=0, \quad a_{0}=\frac{2}{\sqrt{p q}}, \\
b_{0}=\frac{i p}{\sqrt{p q}}, \quad c_{0}=\frac{-i q}{\sqrt{p q}}, \\
a_{1}=\frac{p q_{x}-p_{x} q}{2(1-p q)^{3 / 2}} i, \quad b_{1}=\frac{p_{x}}{2(1-p q)^{3 / 2}}, \\
c_{1}=\frac{-q_{x}}{2(1-p q)^{3 / 2}} .
\end{gathered}
$$

In order to obtain the Hamiltonian function of the WKI soliton hierarchy, we use the trace identity [8]:

$$
\frac{\delta}{\delta u_{i}}\left\langle V, \frac{\partial U}{\partial \lambda}\right\rangle=\left(\lambda^{-r} \frac{\partial}{\partial \lambda} \lambda^{r}\right)\left\langle V, \frac{\partial U}{\partial u_{i}}\right\rangle .
$$

Through the calculating, we have

$$
\begin{aligned}
&\left\langle V, \frac{\partial U}{\partial \lambda}\right\rangle=\operatorname{tr}\left(V, \frac{\partial U}{\partial \lambda}\right) \\
&=-2 \lambda a+2 \lambda p q a+q b_{x}+p c_{x}, \\
&\left\langle V, \frac{\partial U}{\partial q}\right\rangle=\operatorname{tr}\left(V \frac{\partial U}{\partial q}\right)=\lambda^{2} q a+\lambda c_{x}, \\
&\left\langle V, \frac{\partial U}{\partial r}\right\rangle=\lambda^{2} p a+\lambda b_{x} .
\end{aligned}
$$

Therefore we obtain this hierarchy by using the trace identity (7):

$$
\left(\begin{array}{c}
\frac{\delta}{\delta p} \\
\frac{\delta}{\delta q}
\end{array}\right)\left(-i a_{m}+q b_{m}-p c_{m}\right)=(r-m)\left(\begin{array}{c}
-c_{m} \\
b_{m}
\end{array}\right)
$$

When $m=0, r=1$ in (9), we obtain the Hamiltonian structure

$$
\left(\begin{array}{c}
-c_{m} \\
b_{m}
\end{array}\right)=H_{m}\left(\begin{array}{c}
\frac{\delta}{\delta p} \\
\frac{\delta}{\delta q}
\end{array}\right)
$$

with

$$
H_{m}=\frac{i a_{m}-q b_{m}+p c_{m}}{m-1} .
$$

However $m=1$; the $H_{1}$ is meaningless, so we define $H_{1}$ from (11):

$$
H_{1}=\frac{p q_{x}-p_{x} q}{2 p q}\left(1-\frac{1}{\sqrt{1-p q}}\right) .
$$

The soliton WKI equation hierarchy is given rise to as follows:

$$
\begin{aligned}
u_{t}=\left(\begin{array}{l}
p_{t} \\
q_{t}
\end{array}\right) & =\left(\begin{array}{l}
b_{m x x} \\
c_{m x x}
\end{array}\right)=J\left(\begin{array}{c}
-c_{m} \\
b_{m}
\end{array}\right), \\
J & =\left(\begin{array}{cc}
0 & \partial^{2} \\
-\partial^{2} & 0
\end{array}\right),
\end{aligned}
$$


and the recursive operator $L$ and recurrence relation are presented:

$$
\begin{gathered}
\left(\begin{array}{c}
-c_{m+1} \\
b_{m+1}
\end{array}\right)=L\left(\begin{array}{c}
-c_{m} \\
b_{m}
\end{array}\right), \\
L=\left(\begin{array}{cc}
-\frac{i q}{4 \sqrt{1-p q}} \partial^{-1} \frac{p}{\sqrt{1-p q}} \partial^{2}-\frac{i}{2} \partial & \frac{i q}{4 \sqrt{1-p q}} \partial^{-1} \frac{q}{\sqrt{1-p q}} \partial^{2} \\
-\frac{i p}{4 \sqrt{1-p q}} \partial^{-1} \frac{p}{\sqrt{1-p q}} \partial^{2} & \frac{i p}{4 \sqrt{1-p q}} \partial^{-1} \frac{q}{\sqrt{1-p q}} \partial^{2}+\frac{i}{2} \partial
\end{array}\right) .
\end{gathered}
$$

Specially, we consider a case of the WKI equation and its Hamiltonian function:

$$
u_{t}=J\left(\begin{array}{c}
-c_{m} \\
b_{m}
\end{array}\right)=J L^{m}\left(\begin{array}{c}
\frac{i q}{\sqrt{p q}} \\
\frac{i p}{\sqrt{p q}}
\end{array}\right)=J \frac{\delta}{\delta u_{i}} H_{m} .
$$

\section{The Integrable Couplings and Its Hamiltonian Structure}

Integrable coupling system is an interesting and important topic in soliton theory. The theory of integrable couplings brings other interesting results such as Lax pairs of block form and several spectral parameters, integrable constrained flows with higher multiplicity, local bi-Hamiltonian structures in higher dimensions, and hereditary recursion operators of higher order.

Based on the theory of Ma's design of the integrable couplings, a new integrable coupling of WKI soliton hierarchy and its Hamiltonian structure are obtained. We select $\bar{U}$ and $\bar{V}$ as follows:

$$
\begin{aligned}
& \bar{U}(\bar{u}, \lambda)=\left(\begin{array}{ccccc}
-2 i \lambda & p \lambda & 0 & r \lambda & 0 \\
2 q \lambda & 0 & 2 p \lambda & s \lambda & r \lambda \\
0 & q \lambda & 2 i \lambda & 0 & s \lambda \\
0 & 0 & 0 & -i \lambda & p \lambda \\
0 & 0 & 0 & q \lambda & i \lambda
\end{array}\right) \\
& \bar{u}=\left(\begin{array}{c}
p \\
q \\
r \\
s
\end{array}\right), \\
& \bar{V}=\left(\begin{array}{ccccc}
-2 i \lambda a & p \lambda a+b_{x} & 0 & f \lambda & 0 \\
2 \lambda q a+2 c_{x} & 0 & 2 p \lambda a+2 b_{x} & g \lambda & f \lambda \\
0 & q \lambda a+c_{x} & 2 i \lambda a & 0 & g \lambda \\
0 & 0 & 0 & -2 i \lambda a & p \lambda a+b_{x} \\
0 & 0 & 0 & q \lambda a+c_{x} & i \lambda a
\end{array}\right) .
\end{aligned}
$$

From the stationary zero curvature equation

$$
\bar{V}_{x}=[\bar{U}, \bar{V}],
$$

it gives rise to

$$
\begin{aligned}
& -i a_{x}=p c_{x}-q b_{x}, \\
& \lambda p a+b_{x}=-2 i \lambda b, \\
& \lambda q a+c_{x}=2 i \lambda c,
\end{aligned}
$$

$$
\begin{gathered}
f_{x}=-i r \lambda a-p s \lambda a-s b_{x}-i \lambda f+p \lambda g \\
g_{x}=i s \lambda a-q r \lambda a-r c_{x}+q \lambda f+i \lambda g .
\end{gathered}
$$

Setting

$$
\begin{gathered}
a=\sum_{m=1}^{\infty} a_{m} \lambda^{-m}, \quad b=\sum_{m=1}^{\infty} b_{m} \lambda^{-m}, \quad c=\sum_{m=1}^{\infty} c_{m} \lambda^{-m}, \\
f=\sum_{m=1}^{\infty} f_{m} \lambda^{-m}, \quad g=\sum_{m=1}^{\infty} g_{m} \lambda^{-m}
\end{gathered}
$$


by using of the stationary zero curvature equation (17), we obtain the extended recurrence relations

$$
\begin{gathered}
-i a_{m x}=p c_{m x}-q b_{m x}, \\
p a_{m+1}+b_{m x}=-2 i b_{m+1}, \\
q a_{m+1}+c_{m x}=2 i c_{m+1}, \\
f_{m x}=-i r a_{m+1}-p s a_{m+1}-s b_{m x}-i f_{m+1}+p g_{m+1}, \\
g_{m x}=i s a_{m+1}-q r a_{m+1}-r c_{m x}+q f_{m+1}+i g_{m+1},
\end{gathered}
$$

or

$$
\begin{gathered}
a_{m+1}=\frac{1}{2 \sqrt{1-p q}} \partial^{-1}\left(\frac{q}{\sqrt{1-p q}} b_{m x x}+\frac{p}{\sqrt{1-p q}} c_{m x x}\right), \\
b_{m+1}=\frac{i p}{2} a_{m+1}+\frac{i}{2} b_{m x} \\
c_{m+1}=-\frac{i q}{2} a_{m+1}-\frac{i}{2} c_{m x} \\
f_{m+1}=-r a_{m+1}+i p s a_{m+1}+i s b_{m x}+i f_{m x}-i p g_{m+1} \\
g_{m+1}=-i g_{m x}-s a_{m+1}-i q r a_{m+1}-i r c_{m x}+i q f_{m+1} .
\end{gathered}
$$

And the initial value is given as follows:

$$
\begin{gathered}
(2-2 p q) a_{0 x}-(p q)_{x} a_{0}=0, \\
a_{0}=\frac{2}{\sqrt{p q}}, \quad b_{0}=\frac{i p}{\sqrt{p q}}, \\
c_{0}=\frac{-i q}{\sqrt{p q}}, \quad f_{0}=\frac{4 i p s-2 r-2 p q r}{(1-p q)^{3 / 2}}, \\
g_{0}=\frac{(4 q r-2 s-2 p q s}{(1-p q)^{3 / 2}}, \quad a_{1}=\frac{p q_{x}-p_{x} q}{2(1-p q)^{3 / 2}} i, \\
b_{1}=\frac{p_{x}}{2(1-p q)^{3 / 2}}, \quad c_{1}=\frac{-q_{x}}{2(1-p q)^{3 / 2}}, \\
f_{1}=\left(-10 i p p_{x} q^{2} r+3 p p_{x} q s-10 p^{2} p_{x} q^{2} s+11 i p_{x} q r\right. \\
-2 i p^{2} q q_{x} r+9 p^{2} q_{x} s-10 p^{3} q^{3} q_{x} s-i p q_{x} r \\
-8 i p q_{x} r_{x}-4 p s+4 p^{2} q s_{x}+10 p_{x} s+4 i r_{x} \\
\left.+4 i p^{2} q^{2} r_{x}+4 p^{2} q s+4 p^{3} q^{2} s_{x}\right) \frac{1}{2(1-p q)^{5 / 2}} \\
+4 p q^{2} r_{x}+2 q r_{x}-i s_{x}-2 i p^{2} q^{2} s_{x} \\
g_{1}=\left(p_{x} q^{2} r+2 i p p_{x} q s+i p p_{x} q^{2} s-3 p^{2} p_{x} q^{2} r-6 p q_{x} s\right. \\
+4 i p^{2} q q_{x} s-p^{2} q^{2} q_{x} r+6 q_{x} r+4 i p q s_{x} \\
2(1-p q)^{5 / 2}
\end{gathered}
$$

The Hamiltonian structures of the integrable couplings are obtained by using the quadratic identity. In comparison with the prevenient theoretical investigation, we regard our investigation as the generalization of the integrable couplings and Hamiltonian structures of the WKI hierarchy in soliton theory. Then we use the variational identity [6-10], which can generate the coupling Hamiltonian structure. The variational identity is considered as follows [6]:

$$
\begin{gathered}
\frac{\delta}{\delta u_{i}} \int\left\langle\bar{V}, \frac{\partial \bar{U}}{\partial \lambda}\right\rangle d_{x}=\left(\lambda^{-r} \frac{\partial}{\partial \lambda} \lambda^{r}\right)\left\langle\bar{V}, \frac{\partial \bar{U}}{\partial u_{i}}\right\rangle, \\
r=\frac{\lambda}{2} \frac{d}{d_{\lambda}} \ln \left|\operatorname{tr}\left(\bar{V}^{2}\right)\right|
\end{gathered}
$$

where the operation relations are defined by

$$
\begin{aligned}
M(a, b, c, f, g) & =\left(\begin{array}{ccccc}
2 a & b & 0 & f & g \\
2 c & 0 & 2 b & g & f \\
0 & c & -2 a & 0 & g \\
0 & 0 & 0 & a & b \\
0 & 0 & 0 & c & -a
\end{array}\right), \\
M\left(a^{\prime}, b^{\prime}, c^{\prime}, f^{\prime}, g^{\prime}\right) & =\left(\begin{array}{ccccc}
2 a^{\prime} & b^{\prime} & 0 & f^{\prime} & g^{\prime} \\
2 c^{\prime} & 0 & 2 b^{\prime} & g^{\prime} & f^{\prime} \\
0 & c^{\prime} & -2 a^{\prime} & 0 & g^{\prime} \\
0 & 0 & 0 & a^{\prime} & b^{\prime} \\
0 & 0 & 0 & c^{\prime} & -a^{\prime}
\end{array}\right),
\end{aligned}
$$

and the $\langle$,$\rangle is defined as follows:$

$$
\begin{gathered}
\left\langle M(a, b, c, f, g), M\left(a^{\prime}, b^{\prime}, c^{\prime}, f^{\prime}, g^{\prime}\right)\right\rangle \\
=(a, b, c, f, g) F\left(a^{\prime}, b^{\prime}, c^{\prime}, f^{\prime}, g^{\prime}\right)^{T} \\
=\eta_{1}\left(a a^{\prime}+\frac{1}{2} b c^{\prime}+\frac{1}{2} c b^{\prime}\right) \\
+\eta_{2}\left(b g^{\prime}-c f^{\prime}-f c^{\prime}+g b^{\prime}\right),
\end{gathered}
$$

and the transformation matrix is presented:

$$
F=\left(\begin{array}{ccccc}
\eta_{1} & 0 & 0 & 0 & 0 \\
0 & 0 & \frac{1}{2} \eta_{1} & 0 & \eta_{2} \\
0 & \frac{1}{2} \eta_{1} & 0 & -\eta_{2} & 0 \\
0 & 0 & -\eta_{2} & 0 & 0 \\
0 & \eta_{2} & 0 & 0 & 0
\end{array}\right)
$$


According to (24), (25), and (26), we obtain the equations

$$
\begin{gathered}
\left\langle\bar{V}, \frac{\partial \bar{U}}{\partial \lambda}\right\rangle=\eta_{1}(-\lambda a-i q \lambda b+i p \lambda c) \\
+\eta_{2}(p \lambda g-q \lambda f-2 i s \lambda b-2 i r \lambda c) \\
\left\langle\bar{V}, \frac{\partial \bar{U}}{\partial p}\right\rangle=\eta_{1}\left(i \lambda^{2} c\right)+\eta_{2}\left(\lambda^{2} g\right) \\
\left\langle\bar{V}, \frac{\partial \bar{U}}{\partial q}\right\rangle=-\eta_{1}\left(i \lambda^{2} b\right)-\eta_{2}\left(\lambda^{2} f\right) \\
\left\langle\bar{V}, \frac{\partial \bar{U}}{\partial r}\right\rangle=-\eta_{2}\left(2 i \lambda^{2} c\right) \\
\left\langle\bar{V}, \frac{\partial \bar{U}}{\partial s}\right\rangle=-\eta_{2}\left(2 i \lambda^{2} b\right)
\end{gathered}
$$

Based on the variational identity, we obtain the following system:

$$
\begin{gathered}
\left(\begin{array}{c}
\frac{\delta}{\delta p} \\
\frac{\delta}{\delta q} \\
\frac{\delta}{\delta r} \\
\frac{\delta}{\delta s}
\end{array}\right) \int\left[\eta_{1}\left(-a_{m}-i q b_{m}+i p c_{m}\right)\right. \\
=(r-m+1)\left(\begin{array}{c}
\eta_{1}\left(i c_{m}\right)+\eta_{2} g_{m} \\
-\eta_{1}\left(i b_{m}\right)-\eta_{2} f_{m} \\
-\eta_{2}\left(2 i c_{m}\right) \\
-\eta_{2}\left(2 i b_{m}\right)
\end{array}\right), \\
\left.+\eta_{2}\left(p g_{m}-q f_{m}-2 i s b_{m}-2 i r c_{m}\right)\right] d_{x}
\end{gathered}
$$

in which we calculate $r$ by

$$
\begin{aligned}
r & =\frac{\lambda}{2} \frac{d}{d_{\lambda}} \ln \left|\operatorname{tr}\left(\bar{V}^{2}\right)\right| \\
& =\frac{\eta_{1}\left(-\lambda_{2} a_{2}+4 \lambda^{2} b c\right)+\eta_{2}(-4 i \lambda g b-4 i \lambda f c)}{\eta_{1}\left(-\lambda_{2} a_{2}+4 \lambda^{2} b c\right)+\eta_{2}(-4 i \lambda g b-4 i \lambda f c)} \\
& =1 .
\end{aligned}
$$

The Hamiltonian function of coupling WKI equation hierarchy is presented:

$$
\begin{gathered}
\frac{\delta}{\delta \bar{u}} \overline{H_{m}}=\left(\begin{array}{c}
i \eta_{1} c_{m}+\eta_{2} g_{m} \\
-i \eta_{1} b_{m}-\eta_{2} f_{m} \\
-2 i \eta_{2} c_{m} \\
-2 i \eta_{2} b_{m}
\end{array}\right), \\
\overline{H_{m}}=\int\left[\left(\eta_{1}\left(-a_{m}-i q b_{m}+i p c_{m}\right)\right.\right. \\
\left.+\eta_{2}\left(p g_{m}-q f_{m}-2 i s b_{m}-2 i r c_{m}\right)\right) \\
\left.\times(2-m)^{-1}\right] d_{x} .
\end{gathered}
$$

Based on the Tu method, it gives rise to the integrable couplings of WKI equation hierarchy:

$$
\begin{gathered}
\bar{U}_{t}=\left(\begin{array}{l}
p \\
q \\
r \\
s
\end{array}\right)_{t}=\left(\begin{array}{c}
b_{m x x} \\
c_{m x x} \\
f_{m x}+s b_{m x} \\
g_{m x}+r c_{m x}
\end{array}\right)=\bar{J}\left(\begin{array}{c}
-c_{m} \\
b_{m} \\
-g_{m} \\
f_{m}
\end{array}\right), \\
\bar{J}=\left(\begin{array}{cccc}
0 & \partial^{2} & 0 & 0 \\
-\partial^{2} & 0 & 0 & 0 \\
0 & s \partial & 0 & \partial \\
-r \partial & 0 & -\partial & 0
\end{array}\right), \\
\bar{U}_{t}=\left(\begin{array}{c}
p \\
q \\
r \\
s
\end{array}\right)_{t}=\bar{J}\left(\begin{array}{c}
-c_{m} \\
b_{m} \\
-g_{m} \\
f_{m}
\end{array}\right)=\bar{J} M \frac{\delta}{\delta \bar{u}} \overline{H_{m}}
\end{gathered}
$$

with

$$
\begin{aligned}
& M \frac{\delta}{\delta \bar{u}} \overline{H_{m}}=\left(\begin{array}{c}
-c_{m} \\
b_{m} \\
-g_{m} \\
f_{m}
\end{array}\right) \text {, } \\
& M=\left(\begin{array}{cccc}
0 & 0 & -\frac{i}{2 \eta_{2}} & 0 \\
0 & 0 & 0 & \frac{i}{2 \eta_{2}} \\
-\frac{1}{\eta_{2}} & 0 & -\frac{\eta_{1}}{2 \eta_{2}} & 0 \\
0 & -\frac{1}{\eta_{2}} & 0 & \frac{\eta_{1}}{2 \eta_{2}}
\end{array}\right) \text {, }
\end{aligned}
$$

where $\bar{J}$ is Hamiltonian operator.

According to (20), the recursive operator $\bar{L}$ and the recursive relation are presented as follows:

$$
\begin{gathered}
\left(\begin{array}{c}
-c_{m+1} \\
b_{m+1} \\
-g_{m+1} \\
f_{m+1}
\end{array}\right)=\bar{L}\left(\begin{array}{c}
-c_{m} \\
b_{m} \\
-g_{m} \\
f_{m}
\end{array}\right), \\
\bar{L}=\left(\begin{array}{cccc}
\overline{L_{11}} & \overline{L_{12}} & 0 & 0 \\
\overline{L_{21}} & \overline{L_{22}} & 0 & 0 \\
\overline{L_{31}} & \overline{L_{32}} & -\frac{i \partial}{1-p q} & \frac{q \partial}{1-p q} \\
\overline{L_{41}} & \overline{L_{42}} & \frac{p \partial}{1-p q} & \frac{i \partial}{1-p q}
\end{array}\right),
\end{gathered}
$$

with

$$
\begin{gathered}
\overline{L_{11}}=-\frac{i q}{4 \sqrt{1-p q}} \partial^{-1} \frac{p}{\sqrt{1-p q}} \partial^{2}-\frac{i}{2} \partial, \\
\overline{L_{12}}=\frac{i q}{4 \sqrt{1-p q}} \partial^{-1} \frac{q}{\sqrt{1-p q}} \partial^{2},
\end{gathered}
$$




$$
\begin{aligned}
& \overline{L_{21}}=-\frac{i p}{4 \sqrt{1-p q}} \partial^{-1} \frac{p}{\sqrt{1-p q}} \partial^{2}, \\
& \overline{L_{22}}=\frac{i p}{4 \sqrt{1-p q}} \partial^{-1} \frac{q}{\sqrt{1-p q}} \partial^{2}+\frac{i}{2} \partial \text {, } \\
& \overline{L_{31}}=\frac{1}{1-p q}\left[(-s-2 i q r-p q s) \frac{1}{2 \sqrt{1-p q}} \partial^{-1}\right. \\
& \left.\times \frac{p}{\sqrt{1-p q}} \partial^{2}-i r \partial\right], \\
& \overline{L_{32}}=\frac{1}{1-p q}\left[(s+2 i q r+p q s) \frac{1}{2 \sqrt{1-p q}} \partial^{-1}\right. \\
& \left.\times \frac{q}{\sqrt{1-p q}} \partial^{2}+q s \partial\right], \\
& \overline{L_{41}}=\frac{1}{1-p q}\left[(r-2 i p s+p q r) \frac{1}{2 \sqrt{1-p q}} \partial^{-1}\right. \\
& \left.\times \frac{p}{\sqrt{1-p q}} \partial^{2}+p r \partial\right], \\
& \overline{L_{42}}=\frac{1}{1-p q}\left[(-r+2 i q s-p q r) \frac{1}{2 \sqrt{1-p q}} \partial^{-1}\right. \\
& \left.\times \frac{q}{\sqrt{1-p q}} \partial^{2}+i s \partial\right] \text {. }
\end{aligned}
$$

A complex integrable coupling and its Hamiltonian structure of WKI hierarchy are generated as follows:

$$
\begin{aligned}
\bar{U}_{t} & =\left(\begin{array}{l}
p \\
q \\
r \\
s
\end{array}\right)_{t}=\bar{J}\left(\begin{array}{c}
-c_{m} \\
b_{m} \\
-g_{m} \\
f_{m}
\end{array}\right)=\bar{J} \bar{L}\left(\begin{array}{c}
-c_{m-1} \\
b_{m-1} \\
-g_{m-1} \\
f_{m-1}
\end{array}\right) \\
& =\bar{J} \bar{L}^{m}\left(\begin{array}{c}
-c_{0} \\
\frac{i q}{\sqrt{p q}} \\
-g_{0} \\
f_{0}
\end{array}\right)=\bar{J} \bar{L}^{m}\left(\begin{array}{c}
\frac{i p}{\sqrt{p q}} \\
\frac{4 i q r+2 s+2 p q s}{(1-p q)^{3 / 2}} \\
\frac{4 i p s-2 r-2 p q r}{(1-p q)^{3 / 2}}
\end{array}\right) .
\end{aligned}
$$

The hierarchy (35) is derived from the zero-curvature equation; therefore it is integrable in the Lax sense. Again taking $r=s=0, \bar{J}$ and $\bar{L}$ are reduced to $J$ and $L$, respectively. According to the definition of integrable coupling, we find that (35) is the integrable coupling system of the WKI equation hierarchy (15).

The WKI integrable equation hierarchy with variable spectral parameters is presented. Then, a technically feasible way to construct integrable couplings of soliton equations has been presented by using the Lie algebras of block matrix, and the Hamiltonian structures of the integrable coupling of WKI hierarchy are obtained by using the variational identity. A kind of higher-dimensional Lie algebras is given, which can be used to directly construct integrable couplings of the soliton integrable systems. We have derived a new integrable coupling and its Hamiltonian structure of the WKI equation hierarchy.

\section{Conflict of Interests}

The authors declare that there is no conflict of interests regarding the publication of this paper.

\section{Acknowledgments}

This work was supported by the Natural Science Foundation of Liaoning Province, China (Grant no. 2013020056), and Project supported by the National Natural Science Foundation of China (Grant no. 11301349).

\section{References}

[1] A. Pickering, "A new truncation in Painlevé analysis," Journal of Physics A: Mathematical and General, vol. 26, no. 17, pp. 43954405, 1993.

[2] J.-F. Zhang, "Multiple soliton solutions of the dispersive longwave equations," Chinese Physics Letters, vol. 16, no. 1, pp. 4-5, 1999.

[3] E. Fan and H. Zhang, "New exact solutions to a solutions to a system of coupled KdV equations," Physics Letters A, vol. 245, no. 5, pp. 389-392, 1998.

[4] S. Y. Lou, "Obtaining higher-dimensional integrable models by means of Miura type noninvertible transformations," Acta Physica Sinica, vol. 49, no. 9, pp. 1657-1662, 2000 (Chinese).

[5] W. X. Ma and B. Fuchssteiner, "Integrable theory of the perturbation equations," Chaos, Solitons and Fractals, vol. 7, no. 8, pp. 1227-1250, 1996.

[6] W. X. Ma, "Integrable couplings and matrix loop algebra," in Nonlinear and Modern Mathematical Physics, W. X. Ma and D. Kaup, Eds., vol. 1562 of AIP Conference Proceedings, pp. 105-122, American Institute of Physics, Melville, NY, USA, 2013.

[7] W.-X. Ma, "Enlarging spectral problems to construct integrable couplings of soliton equations," Physics Letters A, vol. 316, no. 1-2, pp. 72-76, 2003.

[8] W.-X. Ma, "Integrable couplings of vector AKNS soliton equations," Journal of Mathematical Physics, vol. 46, no. 3, Article ID 033507, 2005.

[9] Y. Zhang and H. Zhang, "A direct method for integrable couplings of TD hierarchy," Journal of Mathematical Physics, vol. 43, no. 1, pp. 466-472, 2002.

[10] Y. Zhang and H.-W. Tam, "The multi-component KdV hierarchy and its multi-component integrable coupling system," Chaos, Solitons and Fractals, vol. 23, no. 2, pp. 651-655, 2005.

[11] W. X. Ma, “On the two sorts of Hamiltonian operators," Journal of Mathematical Physics, vol. 43, pp. 1408-1421, 2002.

[12] L. Frappat, A. Sciarrino, and P. Sorba, Dictionary on Lie Algebras and Superalgebras, Academic Press, San Diego, Calif, USA, 2000. 
[13] S. Yu. Sakovich, "On integrability of a $(2+1)$-dimensional perturbed KdV equation," Journal of Nonlinear Mathematical Physics, vol. 5, no. 3, pp. 230-233, 1998.

[14] S. Yu. Sakovich, "Coupled KdV equations of Hirota-Satsuma type," Journal of Nonlinear Mathematical Physics, vol. 6, no. 3, pp. 255-262, 1999.

[15] W.-X. Ma, "A spectral problem based on $s o(3, R)$ and its associated commuting soliton equations," Journal of Mathematical Physics, vol. 54, no. 10, Article ID 103509, 2013.

[16] E. Fan, "Integrable evolution systems based on GerdjikovIvanov equations, bi-Hamiltonian structure, finite-dimensional integrable systems and $N$-fold Darboux transformation," Journal of Mathematical Physics, vol. 41, no. 11, pp. 7769-7782, 2000.

[17] W.-X. Ma, X.-X. Xu, and Y. Zhang, "Semidirect sums of Lie algebras and discrete integrable couplings," Journal of Mathematical Physics, vol. 47, no. 5, Article ID 053501, 2006.

[18] T. Tsuchida and M. Wadati, "New integrable systems of derivative nonlinear Schrödinger equations with multiple components," Physics Letters A, vol. 257, no. 1-2, pp. 53-64, 1999.

[19] T. Tsuchida and M. Wadati, "Multi-field integrable systems related to WKI-type eigenvalue problems," Journal of the Physical Society of Japan, vol. 68, no. 7, pp. 2241-2245, 1999.

[20] B. Fuchssteiner, Coupling of Completely Integrable Systems, Kluwer, Dordrecht, The Netherlands, 1993.

[21] C. H. Gu, H. S. Hu, and Z. X. Zhou, Soliton Theory and Its Application, Zhejiang Publishing House of Science and Technology, 1990.

[22] X. B. Hu, "A powerful approach to generate new integrable systems," Journal of Physics A, vol. 27, no. 7, pp. 2497-2514, 1994.

[23] Y. Zhang, X. Guo, and H. Tam, "A new Lie algebra, a corresponding multi-component integrable hierarchy and an integrable coupling," Chaos, Solitons \& Fractals, vol. 29, no. 1, pp. 114-124, 2006. 


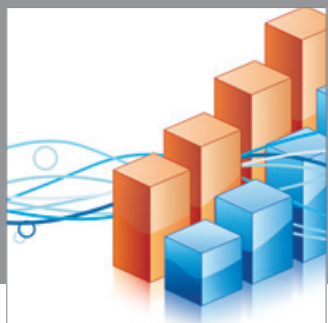

Advances in

Operations Research

mansans

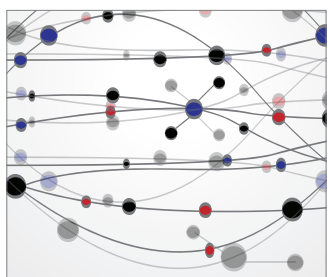

The Scientific World Journal
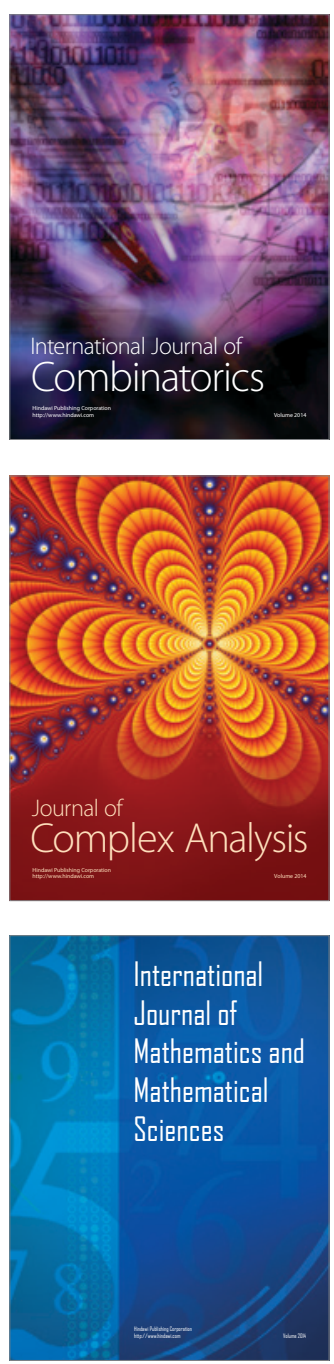
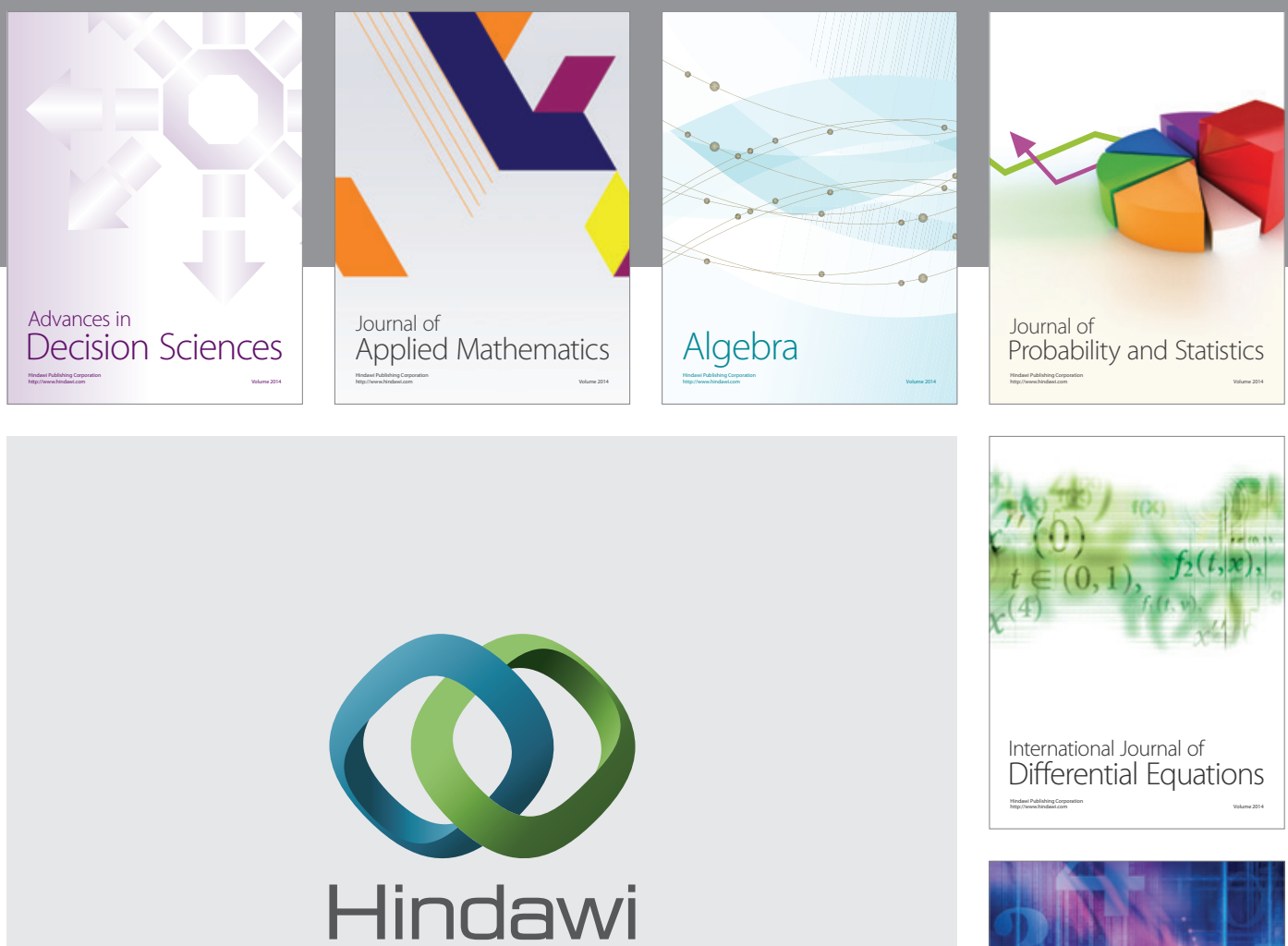

Submit your manuscripts at http://www.hindawi.com
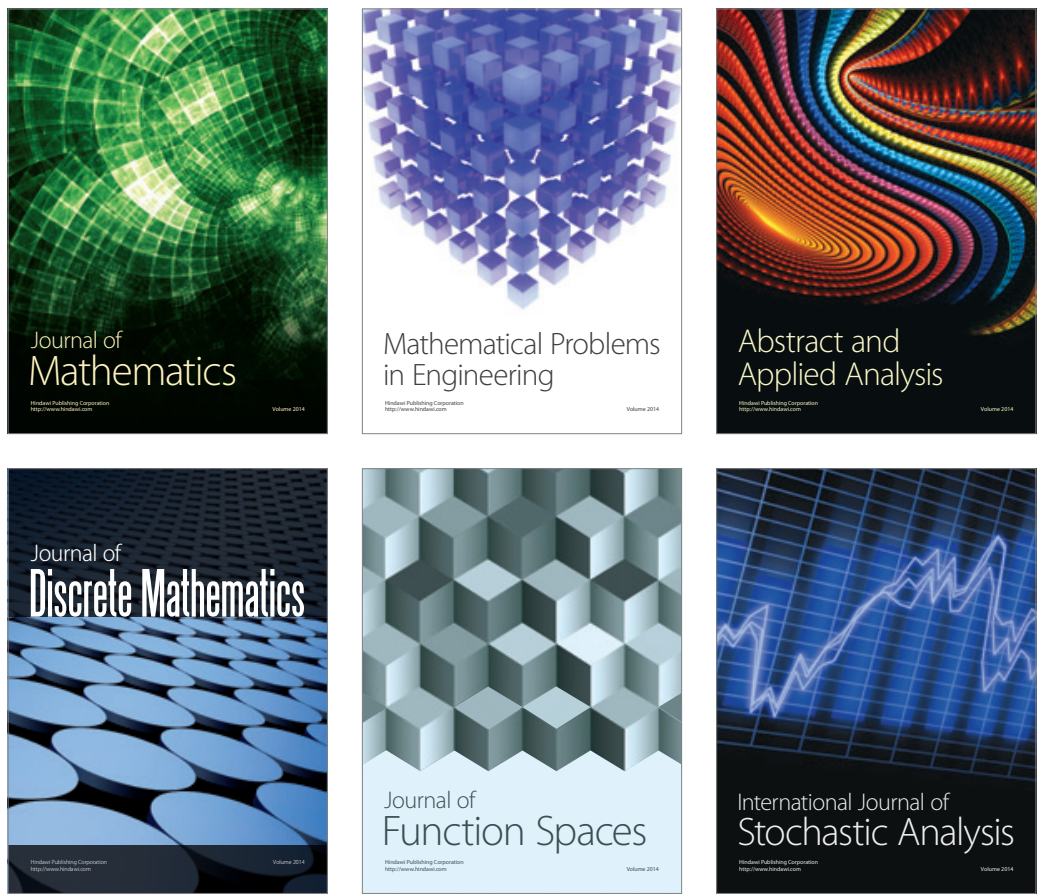

Journal of

Function Spaces

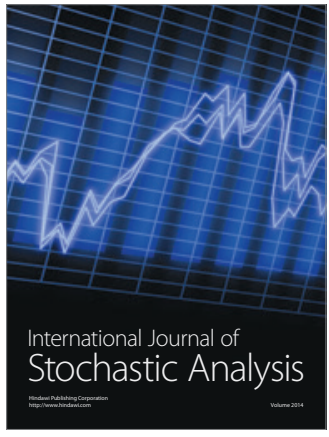

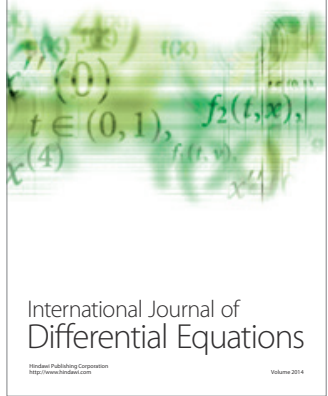
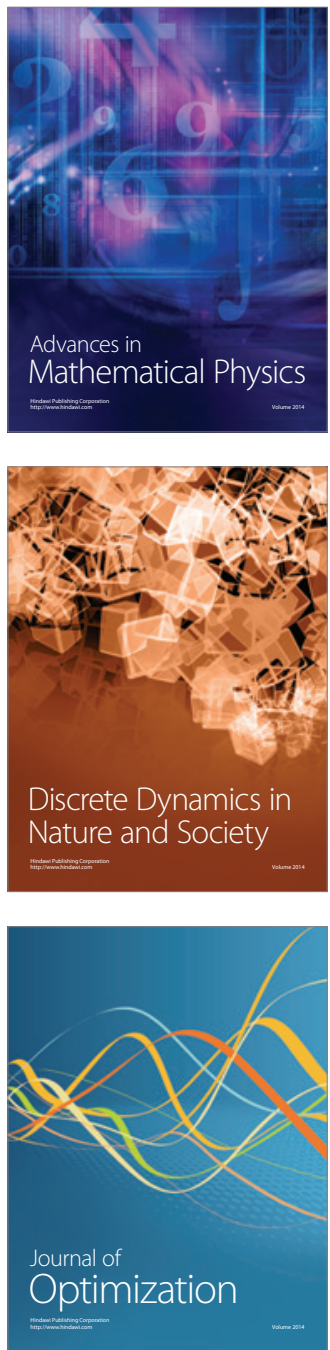